\title{
SOME SPECTRAL PROPERTIES OF AN OPERATOR ASSOCIATED WITH A PAIR OF NONNEGATIVE MATRICES $\left({ }^{1}\right)$
}

\author{
BY \\ M. V. $\left.\operatorname{MENON(}{ }^{2}\right)$
}

\begin{abstract}
An operator-in general nonlinear-associated with a pair of nonnegative matrices, is defined and some of its spectral properties studied. If the pair of matrices are a square matrix $A$ and the identity matrix of the same order, the operator reduces to the linear operator $A$. The results obtained include generalizations of one of the principal conclusions of the theorem of Perron-Frobenius.
\end{abstract}

1. Introduction. Let $A_{m \times n}$ and $B_{m \times n}$ be two nonnegative matrices, i.e., matrices whose entries are nonnegative real numbers. It is assumed that of $A$ or column of $B$ consists entirely of zeros. $r^{(m)}=\left\{r_{1}, \ldots, r_{m}\right\}$ and $c^{(n)}=\left\{c_{1}, \ldots, c_{n}\right\}$ are sets of positive numbers. When will there exist diagonal matrices $D_{m \times m}$ and $E_{n \times n}$, and a positive number $\theta$, such that $D A E$ has its row-sums equal to the $r_{i}$ and $\theta D B E$ has its column-sums equal to the $c_{j}$ ?

This question can be reformulated as follows: Let $\mathscr{N}$ denote the first orthant of real Euclidean $n$-space, $\mathscr{M}$ that of real Euclidean $m$-space, and $\mathscr{N}^{0}$ the subset of $\mathscr{N}$ consisting of points all of whose coordinates are positive. Let $x \in \mathscr{N}^{0}$, $x=\left(x_{1}, \ldots, x_{n}\right)$. Regarding $x$ as a column vector, denoting by $(A x)_{i}$ the $i$ th element of $A x$, and letting $u$ stand for $\left(r_{1} /(A x)_{1}, \ldots, r_{m} /(A x)_{m}\right)$, we see that $x \rightarrow u$ is a mapping of $\mathscr{N}^{0}$ into $\mathscr{M}$ and $u \rightarrow\left(c_{1} /\left(B^{T} u\right)_{1}, \ldots, c_{n} /\left(B^{T} u\right)_{n}\right)$ is a mapping of $u$ into $\mathscr{N}^{0}$, and hence we obtain $x \stackrel{T}{\rightarrow}\left(c_{1} /\left(B^{T} u\right)_{1}, \ldots, c_{n} /\left(B^{T} u\right)_{n}\right)$ as a mapping $T=$ $T\left(A, r^{(m)} ; B, c^{(n)}\right)$ of $\mathscr{N}^{0}$ into $\mathscr{N}^{0}$. We extend this map to a map $T$ of $\mathscr{N}$ into $\mathscr{N}$ by continuity, at those points where it cannot be defined as above. The question asked in the preceding paragraph can now be rephrased as follows: Under what conditions does $T$ have a positive eigenvector $x$ associated with a positive eigenvalue $\theta$ ? For if such a $\theta$ and such an $x$ exist, then on taking $E$ to be $\operatorname{diag}\left(x_{1}, \ldots, x_{n}\right)$ and $D$ to be $\operatorname{diag}\left(u_{1}, \ldots, u_{m}\right)$, we see that $D A E$ has row-sums equal to the $r_{i}$ and $\theta D B E$ has column-sums equal to the $c_{j}$. In this paper some of the spectral properties of $T$, and particularly the question posed above, are studied.

The operator $T$ was introduced by us in [3], and it was shown that if either $A$ or $B$ were positive, then $T x=\theta x$ regarded as an equation in $x$ and $\theta, x \in \mathscr{N}, x \neq 0$, $\theta \geqq 0$, has one and only one solution $T x_{0}=\theta_{0} x_{0}$, given by $x_{0} \in \mathscr{N}^{0}$ and $\theta_{0}>0$.

Received by the editors January 1, 1967.

(1) Sponsored by the Mathematics Research Center, United States Army, Madison, Wisconsin, under contract No. DA-31-ARO-D-462.

${ }^{2}$ ) Present address is University of Missouri, Columbia, Missouri. 
That this conclusion holds in the case $A=B$, under the added and necessary assumption that there exists a matrix of the same pattern as $A$, and with row-sums equal to the $r_{i}$ and column-sums equal to the $c_{j}$, was shown in [4] (see also §6). The approach to the problem was the 'matrix-reduction approach' of the first paragraph.

The yet more special case when $A$ and $B$ are not only equal but are also square matrices was treated in [6] using the 'matrix-reduction approach', and in [1] using the 'nonlinear operator approach' of the second paragraph. We refer the reader to [1] for references to other work related to that of this paper.

2. Notation. The definitions and notations $A, B, T$, etc., of $\S 1$ will be used throughout the paper. $x, y, \ldots$ will, unless stated to the contrary, stand for elements of $\mathscr{N}$. Since $T=T\left(A, r^{(m)} ; B, c^{(n)}\right)=T\left(\tilde{A}, 1^{(m)} ; \widetilde{B}, 1^{(n)}\right)$, where $1^{(m)}$ and $1^{(n)}$ are $m$ - and $n$-vectors consisting entirely of unit elements, and $\tilde{A}=\operatorname{diag}\left(1 / r_{1}, \ldots, 1 / r_{m}\right) A$ and $\widetilde{B}=B \operatorname{diag}\left(1 / c_{1}, \ldots, 1 / c_{n}\right)$, we may, and shall, assume in all sections except the last one, that $r^{(m)}=1^{(m)}$ and $c^{(n)}=1^{(n)}$. With this assumption in mind, we write $T=T(A ; B)$.

If $U$ is any continuous operator on $\mathscr{N}$ into $\mathscr{N}$, and $x \in \mathscr{N}$ is given, then the greatest nonnegative number $\lambda$ for which $U x \geqq \lambda x$ holds will be denoted by $\Lambda(x)$. $\Lambda(x)$ thus depends on $U$ even though this is not explicitly indicated in the symbol. As in [2], by the term maximal eigenvalue of $U$ we mean that positive eigenvalue, if any such exists, which is not less than any other positive eigenvalue. We write eigenvector or eigenvalue to mean, in general, a real nonnegative eigenvector or a real, nonnegative eigenvalue.

If $X$ is any $m \times n$ matrix, and $1 \leqq i_{1}, \ldots, i_{r} \leqq m$ and $1 \leqq j_{1}, \ldots, j_{s} \leqq n$, then $X\left[i_{1}, \ldots, i_{r} \mid j_{1}, \ldots, j_{s}\right]$ stands for the submatrix of $X$ determined by the rows $i_{1}, \ldots, i_{r}$ and the columns $j_{1}, \ldots, j_{s}$.

3. General properties of $T$. The following theorem states some obvious properties that $T=T(A ; B)$ possesses. We recall that $A$ is assumed to have no zero rows and $B$ to have no zero columns.

THEOREM 3.1. T maps $\mathscr{N}$ continuously into itself and $\mathscr{N}^{0}$ into itself. $T$ is homogeneous of degree one and is monotonically increasing. If $x \in \mathscr{N}^{0}$, then $x_{i}>y_{i}$, for all $i$, implies that $(T x)_{i}>(T y)_{i}$ for all $i$.

The proof of the next result is also easy, and is given in [5].

THEOREM 3.2. Let $U$ be a continuous operator on $\mathscr{N}$ into $\mathscr{N}$ which is homogeneous of degree one and which is such that $U x \neq 0$ if $x \neq 0$. Then there exists a positive eigenvalue associated with a nonnegative eigenvector.

The next theorem is proved in [1].

THEOREM 3.3. Let $U$ be a continuous operator on $\mathscr{N}$ into $\mathscr{N}$. Then $\Lambda(x)$ is upper semicontinuous on $\mathscr{N}-0$. If $U$ is also homogeneous and there exists an $x$ such that $(U x)_{i}>0$, for all $i$, then there exists a positive number $\rho$ and $u \in \mathscr{N}, u \neq 0$, such that $\rho=\sup \{\Lambda(x) \mid x \neq 0\}=\Lambda(u)$. 
THEOREM 3.4. Let $U$ be a continuous, monotonically increasing operator on $\mathscr{N}$ into $\mathscr{N}$ which is also homogeneous of degree one. Let $U x=\sigma x$ and $U y \geqq \delta y$, and let $y_{i}=0$ whenever $x_{i}=0$. Then $\sigma \geqq \delta$.

Proof. There exists a positive number $\alpha$ such that $\alpha y \leqq x$, and $\alpha y_{i}=x_{i} \neq 0$, for some $i$. But $\alpha y \leqq x \Rightarrow U \alpha y \leqq U x \Rightarrow \alpha \delta y_{i} \leqq \sigma x_{i} \Rightarrow \delta \leqq \sigma$.

COROLlARY 1. Eigenvectors of $U$ with the same pattern of zero and nonzero elements have the same eigenvalues. Hence the number of eigenvalues is finite. (This corollary is also contained in Theorem 2 of [5].)

CoRollary 2. Any positive eigenvector has its eigenvalue not less than the eigenvalue associated with any nonnegative eigenvector.

COROLlARY 3. If a positive eigenvector $x$ exists with associated eigenvalue $\sigma$, then $\sigma=\rho=\sup \{\Lambda(x) \mid x \neq 0\}$.

Because of Theorem 3.1, we see that the preceding two theorems hold for $T$.

THEOREM 3.5. If either $A$ or $B$ is a positive matrix, then $T$ has a unique nonnegative eigenvalue $\rho$ and a unique nonnegative eigenvector $u$. Both of these are indeed positive. Further $\Lambda(u)=\sup \{\Lambda(x) \mid x \neq 0\}=\rho$.

The proof of this result is essentially contained in [3]. All but the last conclusion follows from Theorem 3 of [5] also.

COROllaRY. If $A$ has no zero row and $B$ has no zero column, then $T(A ; B)$ has eigenvalue the positive number $\rho=\sup \{\Lambda(x) \mid x \neq 0\}$. The associated eigenvector $u$ is nonnegative and $\Lambda(u)=\rho$.

The proof is along the same lines as that used for the reducible linear operator in [2, p. 66] and we merely sketch it. Let $T_{\varepsilon}=T\left(A_{\varepsilon} ; B\right)$ where $A_{\varepsilon}$ differs from $A$ only in that all the zero entries of $A$ are replaced by a positive number $\varepsilon$. As $\varepsilon \rightarrow 0$, $T_{\varepsilon} x \rightarrow T x$, uniformly for all $x$ for which $\sum x_{i}=1$. Further, in finding the supremum of $\Lambda(x)$ over all $x \neq 0$, it is sufficient, from the homogeneity of $T$, to take into account all $x$ such that $\sum x_{i}=1$. Now, by the preceding theorem, $T_{\varepsilon}$ has the unique maximal eigenvalue $\rho_{\varepsilon}$, and one shows easily that $\rho_{\varepsilon} \rightarrow \rho$ as $\varepsilon \rightarrow 0$, and if $u_{\varepsilon}$ is the eigenvector of $T_{\varepsilon}$ associated with $\rho_{\varepsilon}$, then as $\varepsilon \rightarrow 0, u_{\varepsilon}$ has a limit point $u$.

N.B. If for each $\varepsilon>0, \rho_{\varepsilon} \gtrless$ a constant, then $\rho \gtrless$ the same constant.

4. Theorem of Perron-Frobenius. Let us observe that if $A$ is a square matrix of order $n$ and $I$ is the identity matrix of the same order then $T(A ; I)$ reduces to the linear operator (represented by) $A$.

One of the principal conclusions of the theorem of Perron-Frobenius is that when $A$, assumed in this section to be a square matrix, is irreducible, it has a unique positive eigenvector associated with a unique positive eigenvalue, the latter being the eigenvalue of maximum modulus. Now, in general, conclusions about the 
spectral characteristics of $A$ must take into account the magnitudes of the entries of $A$. The theorem of Perron-Frobenius shows that some conclusions can be reached taking into account merely the pattern of $A$. However, more light is thrown on the concept of irreducibility if one looks upon it not solely as a statement about the pattern of $A$, but also as one about the pattern of $A$ vis à vis that of the identity matrix I (cf. (2) below). Indeed, as is easily verified, the following statements are equivalent:

(1) $A$ is irreducible.

(2) There does not exist $1 \leqq i_{1}, \ldots, i_{r} \leqq m$ such that for every $1 \leqq j_{1}, \ldots, j_{s} \leqq n$ the following statement holds: $I\left[i_{1}, \ldots, i_{r} \mid j_{1}, \ldots, j_{s}\right]$ is a zero matrix implies $A\left[i_{1}, \ldots, i_{r} \mid j_{1}, \ldots, j_{s}\right]$ is a zero matrix.

(3) There does not exist a vector $x=\left(x_{1}, \ldots, x_{n}\right)$ such that $x_{i_{1}}=\cdots=x_{i_{r}}=0$ and the other $x_{i} \neq 0$ implies $(A x)_{i_{1}}=\cdots=(A x)_{i_{r}}=0$.

Thus (2) may be taken to be the definition of the irreducibility of $A$, and provides the source for our definition of the irreducibility of a matrix with respect to another given in the next section.

Finally, we observe that the imposition of the condition of irreducibility in the theorem of Perron-Frobenius is meant precisely to ensure that (3) holds. It is condition (3) that enables one to reach the conclusions of the theorem about the existence of a positive eigenvalue and an associated positive eigenvector. For, an exceedingly simple argument using the fixed-point theorem (cf. [5] or [3]) shows that $A$ has a nonnegative eigenvector associated with a positive eigenvalue. But (3) guarantees that such an eigenvector must be positive.

The foregoing considerations motivate the next section.

5. Reducibility of one matrix with respect to another. Two $m \times n$ matrices are said to have the same pattern if either of them has a zero in any position when and only when the other has a zero in that position. The composite pattern of a set of $m \times n$ matrices is the pattern of that $m \times n$ matrix which has a zero in any position if and only if all the matrices of the set have zeroes in that position. The pattern of a matrix is subordinate to that of another if the first has zero entries in any position if the second one does. Viewing a row of an $m \times n$ matrix as a $1 \times n$ matrix, we speak of the pattern of a row, and of the composite pattern of a set of rows, etc.

Let $\mathscr{P}$ be the set of composite patterns of all possible collections of the rows of $B$. (Here, the word 'pattern' could be taken, for instance, to mean a $1 \times n$ matrix whose entries are either zeroes or ones.) For $p \in \mathscr{P}$, we define $f(p)$ to be the composite pattern of all those rows of $B$ for which the corresponding rows of $A$ have pattern subordinate to $p$. If there are no rows of $A$ with patterns subordinate to $p$, we define $f(p)$ to be the pattern of the $1 \times n$ matrix whose entries are all zeroes.

Definition. $A$ is reducible with respect to $B$ if there exists $p \in \mathscr{P}$ such that $f(p)=p$, but $p$ is not the pattern either of the $1 \times n$ matrix consisting solely of zeroes or of that consisting solely of ones. 
If $A$ is not reducible with respect to $B$, then $A$ is said to be irreducible with respect to $B$. We say that $T(A ; B)$ is irreducible if $A$ is irreducible with respect to $B$.

N.B. Clearly, if $m=n, A$ has no zero columns and $I$ is the identity matrix of order $n$, then $A$ is reducible with respect to $I$ if and only if $A$ is reducible.

The 'only if' part of this statement is obvious by Theorem 5.1. To prove the 'if' part, suppose that $R$ and $R_{1}$ are subsets of $\{1, \ldots, n\}, R \subset R_{1}, R \neq R_{1}$, with the property that if $x_{i}=0, i \in R$ and $x_{i} \neq 0, i \notin R$, then $(A x)_{i}=0, i \in R_{1}$, and $(A x)_{i} \neq 0$, $i \notin R_{1}$. Because of the assumption that no column of $A$ consists entirely of zeroes, it follows that $R_{1} \neq\{1, \ldots, n\}$. Now, consider $y$, with $y_{i}=0$ if and only if $i \in R_{1}$. Then there exists $R_{2} \supset R_{1}$ such that $(A y)_{i}=0$ if and only if $i \in R_{2}$. If $R_{2}=R_{1}$ the proof is complete. If $R_{2}$ properly contains $R_{1}$, we proceed as above and obtain, in a finite number of steps, a set $R_{u}$ with the following properties. $R_{u}$ is a proper subset of $\{1, \ldots, n\}$ and if $z$ is such that $z_{i}=0$ if and only if $i \in R_{u}$ then $(A z)_{i}=0$ if and only if $i \in R_{u}$.

The definition of the irreducibility of $A$ with respect to $I$ is thus a slight weakening of that of the irreducibility of $A$. We recall that $T(A ; I)$ is the linear operator $A$.

THEOREM 5.1. The following statements are equivalent:

(1) $A$ is irreducible with respect to $B$.

(2) $T(A ; B)$ has the property that for no proper subset $S$ of $\{1, \ldots, n\}$ is it true that $x_{i}=0, i \in S, x_{i} \neq 0, i \notin S$, implies that $(T x)_{i} \neq 0, i \in S$ and $(T x)_{i} \neq 0, i \notin S$.

Proof. If $A$ is reducible with respect to $B$, there exists $p \in \mathscr{P}$ such that $f(p)=p$, and $p$ is the pattern neither of the $1 \times n$ matrix of all zeroes nor of that of all ones. Let $x$ have a pattern which is the complement of $p$. Then, clearly, $x_{i}=(T x)_{i}=0$ if and only if $i \in S$, where $S$ is a proper subset of $\{1, \ldots, n\}$. The proof is completed by reversing the argument.

N.B. The analogue of statement (2) of $\S 4$ is not equivalent to the preceding statements as is shown by the following examples: Let $A$ and $B$ be $3 \times 3$ matrices with $a_{13}=a_{23}=b_{13}=0$, the remaining elements being positive. Then $B[1 \mid 3]$ is the only zero matrix of $B . A[1 \mid 3]$ is also a zero submatrix of $A$. But $A$ is irreducible with respect to $B$.

On the other hand, let $A$ and $B$ be $3 \times 3$ matrices with $a_{13}=a_{23}=a_{32}=b_{13}$ $=b_{21}=b_{23}=0$, the remaining elements being positive. Then $B[1,2 \mid 3]$ is the only zero submatrix of $B$ with elements chosen from the first and second rows of $B$. $A[1,2 \mid 3]$ is also a zero matrix. Here, $A$ is reducible with respect to $B$.

Examples of classes of matrix-pairs, $A$ and $B$, where $A$ is irreducible with respect to $B$ are:

(1) $A$ is irreducible, $B=I_{n \times n}$.

(2) Either $A$ or $B$ is positive.

Examples of classes of matrix-pairs $A$ and $B$, where $A$ is reducible with respect to $B$ are: 
(1) $A$ is reducible, has no zero columns, and $B=I_{n \times n}$.

(2) $A$ and $B$ are of the same pattern, and $A$-and therefore also $B$, - has at least one zero element.

THEOREM 5.2. Let T be irreducible. Then there exists one and only one nonnegative eigenvector $u$ and one and only one nonnegative eigenvalue $\sigma . u$ and $\sigma$ are both positive and $\sigma=\rho$.

Proof. By (2) of Theorem 5.1, any eigenvectors that exist must be positive, and by Corollary 1 to Theorem 3.4, they must have the same eigenvalue. But, the corollary to Theorem 3.5 assures us that there exists the positive eigenvalue $\rho$. Hence, in order to complete the proof, we need to show that if $T x=\rho x$ and $T y=\rho y, x, y$, $>0$, then $y$ is a multiple of $x$.

We denote in what follows, the sets $\{1, \ldots, m\}$ and $\{1, \ldots, n\}$ by $M$ and $N$ respectively. $M_{1}, M_{2}$ will stand for subsets of $M$ and $N_{1}, N_{2}$ for subsets of $N . M_{1}^{\prime}$ will be the complement of $M_{1}$ with respect to $M$ and similar meanings will hold for $M_{2}^{\prime}, N_{1}^{\prime}, N_{2}^{\prime}$.

Let us assume temporarily, that $A$ has no zero columns. Now, there exists $c>0$ such that $c y_{i}<x_{i}, i \in N_{1}$ and $c y_{i}=x_{i}, i \in N_{1}^{\prime}$, where $N_{1}^{\prime}$ is nonnull. If $N_{1}^{\prime}=N$, there is nothing left to prove. Therefore assume that $N_{1}^{\prime}$ is a proper subset of $N$. Let $M_{1}$ consist of all the elements of $M$ for which $A\left[M_{1} \mid N_{1}\right]=0$. Because it has been assumed that $A$ has no zero columns, we have $M_{1} \neq M$. If $M_{1}=\varnothing$, the fact that $B$ has no zero columns will mean that $(T c y)_{i}<(T x)_{i}$ for each $i \in N_{1}^{\prime}$, i.e., $c \rho y_{i}<\rho x_{i}$, $i \in N_{1}^{\prime}$. But $c y_{i}=x_{i} \neq 0, i \in N_{1}^{\prime}$. Thus we have a contradiction. Hence $M_{1}$ is a proper subset of $M$.

If $(T c y)_{i}<(T x)_{i}$ for any $i \in N_{1}^{\prime}$, then we arrive at a contradiction, as in the preceding paragraph. But $(T c y)_{i}=(T x)_{i}$ for each $i \in N_{1}^{\prime}$ only if $B\left[M_{1}^{\prime} \mid N_{1}^{\prime}\right]=0$. Suppose, then, that this is the case.

Now, however, there exists $d>0$ such that $d y_{i}>x_{i}, i \in N_{2}$ and $d y_{i}=x_{i}, i \in N_{2}^{\prime}$. Then $N_{1}^{\prime} \subset N_{2}$, and $N_{2} \neq N$. Let $M_{2}$ be the subset consisting of all elements of $M$ for which $A\left[M_{2} \mid N_{2}\right]=0$. Since $A$ has no zero rows $M_{2} \cap M_{1}=\varnothing$ and hence $M_{2}^{\prime} \supset M_{1}$. It may also be assumed that $M_{2}$ is a proper subset of $M$.

Now if $B\left[M_{2}^{\prime} \mid N_{2}^{\prime}\right] \neq 0$, then there arises a contradiction as before. Suppose, then, that $B\left[M_{2}^{\prime} \mid N_{2}^{\prime}\right]=0$. This implies that $B\left[M_{1} \mid N_{2}^{\prime}\right]=0$. If we now recall the facts that $N_{2}^{\prime} \subset N_{1}$ and that $A\left[M_{1} \mid N_{1}\right]=0$, it follows easily that there is a composite pattern $p$ from among rows $i$ of $B, i \in M_{2}^{\prime}$, such that $f(p)=p$, and furthermore $p$ is not the pattern of a vector consisting solely of zeroes or of one consisting solely of ones. (The definition of $f()$ is given early in this section.) We have thus arrived at the conclusion that $T$ is reducible, contrary to our hypothesis. The proof that $y$ is a multiple of $x$ is now complete for the case where $A$ has no zero columns.

If some of the columns of $A$ consist entirely of zeroes we may, without loss of generality, assume that these are the last $n-p$ columns, where $0<p<n$. Let $A_{1}$ and $B_{1}$ be the matrices obtained from $A$ and $B$ respectively, by omitting the last $n-p$ 
columns for each. $T_{1}=T\left(A_{1} ; B_{1}\right)$ is irreducible since $T$ is. $T_{1}$ has just been shown to possess a unique positive eigenvector $\left(x_{1}, \ldots, x_{p}\right)$ corresponding to a unique positive eigenvalue, which is clearly $\rho$.

Consider a vector $x=\left(x_{1}, \ldots, x_{p}, x_{p+1}, \ldots, x_{n}\right)$. If $x$ is an eigenvector for $T$ with eigenvalue $\rho$, we have in particular, $(T x)_{i}=\rho x_{i}, i>p$. For any such $i,(T x)_{i}$ is a function only of $x_{1}, \ldots, x_{p}$ and hence, is uniquely determined. Thus $x_{i}$ is uniquely determined for $i>p$.

The theorem is now fully proved.

As an obvious corollary to the theorem we have

COROLlaRY. If $A$ is irreducible with respect to $B$, there exists a row-stochastic matrix $A_{1}$, a column-stochastic matrix $A_{2}$, a positive number $\theta$, and two diagonal matrices $D$ and $E$ with positive diagonal entries such that $D A E=A_{1}$ and $\theta D B E=A_{2}$. $A_{1}, A_{2}$ and $\theta$ are uniquely determined. $D$ and $E$ are also uniquely determined up to $a$ scalar multiple.

REMARK. In [5], a continuous, monotone increasing operator $U$ on $\mathscr{N}$ into $\mathscr{N}$ which is homogeneous of degree one is called indecomposable if the following condition is satisfied: The relations $x_{i}=y_{i}, i \in R$, where $R \subset\{1, \ldots, n\}$ and $x_{i}<y_{i}$, $i \notin R$, imply that there exists at least one $i \in R$ for which $(U x)_{i}<(U y)_{i}$.

Taking $U$ to be the operator $T(A ; B)$ we see that indecomposability implies irreducibility. That the reverse implication need not hold is seen by considering the following example: Let $A$ and $B$ be $2 \times 2$ matrices whose only zero elements are $a_{12}$ and $b_{21}$. Let $R=\{1\}$.

6. When $T(A ; B)$ is reducible, general results about its spectrum cannot normally be obtained without taking into account the magnitudes of the elements of $A$ and $B$, and not merely their patterns. The case $A=B$ is, however, an exception to this statement. In a joint paper, Professor Hans Schneider and the author of this paper have obtained necessary and sufficient conditions that $A, r_{1}, \ldots, r_{m}, c_{1}, \ldots, c_{n}$ have to satisfy in order that $T\left(A, r^{(m)} ; B, c^{(n)}\right)$ should have a positive eigenvalue associated with a positive eigenvector. This along with other results will appear elsewhere.

\section{REFERENCES}

1. R. A. Brualdi, S. V. Parter and H. Schneider, The diagonal equivalence of a non-negative matrix to a stochastic matrix, J. Math. Anal. Appl. 16 (1966), 31-50.

2. F. R. Gantmacher, The theory of matrices. II, Chelsea, New York, 1959.

3. M. V. Menon, Reduction of a matrix with positive elements to a doubly stochastic matrix, Proc. Amer. Math. Soc. 18 (1967), 244-247.

4. - Matrix links, an extremisation problem and the reduction of a non-negative matrix to one with prescribed row and column sums, Canad. J. Math. 20 (1968), 225-232.

5. M. Morishima, Generalizations of the Frobenius-Wielandt theorems for non-negative square matrices, J. London Math. Soc. 36 (1961), 211-220.

6. R. Sinkhorn and P. Knopp, Concerning non-negative matrices and doubly stochastic matrices, Pacific J. Math. 21 (1967), 343-348.

UNIVERSITY OF MISSOURI, Columbia, Missouri 